University of Nebraska - Lincoln

DigitalCommons@University of Nebraska - Lincoln

Ralph Skomski Publications

Research Papers in Physics and Astronomy

September 1998

\title{
Higher-order and next-nearest-neighbor Néel anisotropies
}

Y.T. Millev

Max-Planck-Institut für Mathematik in den Naturwissenschaften, Inselstr. 22-26, D-04103 Leipzig,

Germany

Ralph Skomski

University of Nebraska-Lincoln, rskomski2@unl.edu

J. Kirschner

Max-Planck-Institut für Mikrostrukturphysik

Follow this and additional works at: https://digitalcommons.unl.edu/physicsskomski

Part of the Physics Commons

Millev, Y.T.; Skomski, Ralph; and Kirschner, J., "Higher-order and next-nearest-neighbor Néel anisotropies " (1998). Ralph Skomski Publications. 19.

https://digitalcommons.unl.edu/physicsskomski/19

This Article is brought to you for free and open access by the Research Papers in Physics and Astronomy at DigitalCommons@University of Nebraska - Lincoln. It has been accepted for inclusion in Ralph Skomski Publications by an authorized administrator of DigitalCommons@University of Nebraska - Lincoln. 


\title{
Higher-order and next-nearest-neighbor Néel anisotropies
}

\author{
Y. T. Millev* \\ Max-Planck-Institut für Mathematik in den Naturwissenschaften, Inselstr. 22-26, D-04103 Leipzig, Germany
}

R. Skomski and J. Kirschner

Max-Planck-Institut für Mikrostrukturphysik, Weinberg 2, D-06120 Halle, Germany

(Received 8 December 1997; revised manuscript received 17 March 1998)

\begin{abstract}
The problem of higher-order Néel anisotropies is solved by exploiting the addition theorem for spherical functions. A key advantage of the present approach is the orthonormal character of the expansion of the magnetic energy that simplifies the formalism and makes possible the treatment of nonideal morphologies as well. Explicit expressions for second-, fourth-, and sixth-order anisotropies are obtained for ideal bulk of fcc and bec symmetry as well as for (001), (110), and (111) surfaces with nearest-neighbor (NN) Néel interactions. The systematic examination of the pair model involves partition by species of inequivalent sites, interaction spheres, and orders in the multipole expansion. It enables us to to treat also next-nearest-neighbor (NNN) pair interactions to the same high orders as the NN ones. The analysis sheds light onto the peculiar cases of bcc(100) and bcc(111) surfaces where one finds no symmetry breaking (no second-order contributions) with NN interactions only. With the extension to NNN's, it is demonstrated that bcc(111) surfaces exhibit a particularly high symmetry and acquire no second-order anisotropy contributions from NNN interactions, whereas the latter induce a second-order symmetry breaking in the bcc(100) case. [S0163-1829(98)05734-8]
\end{abstract}

\section{INTRODUCTION AND BASICS OF THE MODEL}

Since Néel's pioneering work on surface anisotropy, ${ }^{1}$ the expansion of magnetic anisotropy contributions into Legendre polynomials has become a widely used tool in surface, thin-film, and interface magnetism (see, e.g., Refs. 2-8). There are many successful applications of Neel's theory to scientifically and technologically important problems such as in-plane and out-of-plane anisotropies in low-symmetry surfaces, but most papers are based on lowest-order Néel anisotropies. Higher-order Néel contributions have attracted much less attention, ${ }^{2,6}$ which is largely due to their presumbaly very small magnitude. However, there can be no general justification for the neglection of higher-order anisotropies, particularly since competing anisotropy contributions may give rise to small or zero lowest-order anisotropies. Furthermore, it is difficult to extend first-principles calculations s, $^{2,9}$ to higher-order anisotropy contributions, so that higher-order Néel considerations are a useful complementary tool in anisotropy theory.

The basic assumption of Néel's theory is that the magnetic anisotropy energy of a uniformly magnetized ferromagnet depends on the angle between the uniform magnetization direction and the coordination vectors $\mathbf{r}_{i j}=\mathbf{r}_{j}-\mathbf{r}_{i}$ between atomic moments located at $\mathbf{r}_{i}$ and $\mathbf{r}_{j}$. The total anisotropy results from summing over pair interactions that are cast in the form of a cylindrically symmetric multipole expansion:

$$
u_{i j}=\sum_{l} a_{l}\left(r_{i j}\right) P_{l}\left(\cos \alpha_{i j}\right)
$$

Here, $r_{i j}=\left|\mathbf{r}_{i j}\right|, \alpha_{i j}$ is the angle between $\mathbf{r}_{i j}$ and $\mathbf{M}$ [Fig. 1(a)], while $P_{l}(x)$ are the Legendre polynomials of degree $l$ (see, e.g., Ref. 10). The relevant facts about the special functions used in this paper are summarized in Appendix A. The $l=0$ term is skipped since it is angle independent, while uneven values of $l$ are ruled out by the assumption that the interaction is invariant under reversal of the macroscopic magnetization ( $\mathbf{M} \leftrightarrow-\mathbf{M})$. Consequently, the sums over $l$ below involve positive even values only. The coefficients $a_{l}\left(r_{i j}\right)$ parametrize the atomic origin of the anisotropy in Néel's model. The total interaction energy is

$$
U=\frac{1}{2} \sum_{i \neq j} u_{i j},
$$

where the prefactor takes care about avoiding double counting, while $i$ and $j$ run over all interacting sites. The systematic examination of the pair model of magnetic anisotropy involves partition by species of inequivalent sites, interaction

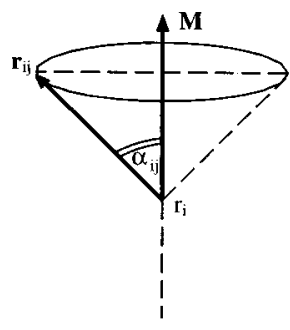

(a)

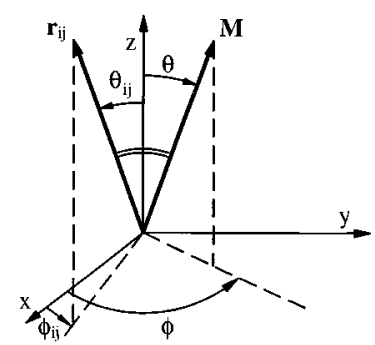

(b)
FIG. 1. (a) The vector connecting sites $\mathbf{i}$ and $\mathbf{j}$ makes an angle $\alpha_{i j}$ with the vector of macroscopic magnetization $\mathbf{M}$. The pair interaction is assumed cylindrically symmetric about $\mathbf{M}$. (b) The vectors from (a) when referred to a specified frame of reference: $\theta_{i j}$ and $\phi_{i j}$ are the polar and azimuthal angles for the pair vector, $\Theta$ and $\Phi$ are the respective ones for $\mathbf{M}$. The connection of these with the angle $\alpha_{i j}$ between the two vectors is provided by the spherical addition theorem that gives rise to the addition theorem for the Legendre functions [cf. Eq. (8)]. 
spheres, and orders in a multipole expansion. In particular, one must always distinguish between local and overall anisotropy coefficients when there are inequivalent magnetic sites. ${ }^{11}$ For this reason we concentrate on the interaction energy per site $i$ :

$$
U(i)=\frac{1}{2} \sum_{j} u_{i j}=\frac{1}{2} \sum_{j} \sum_{l} a_{l}\left(r_{i j}\right) P_{l}\left(\cos \alpha_{i j}\right) .
$$

If we assume that there are $p$ inequivalent sites, then the total magnetic energy per magnetic site can be written as

$$
\frac{U}{N}=\sum_{\mu=1}^{p}\left(\frac{N_{\mu}}{N}\right) U(\mu)
$$

with $U(\mu)$ given by Eq. (3). Here, $N_{\mu}$ is the number of magnetic atoms of the $\mu$ th species [characterized by $U(\mu)$ ] and $N_{1}+N_{2}+\cdots+N_{p}=N$ is their total number. ${ }^{12}$

Changing the order of summation in Eq. (3), which is mathematically harmless as long as one considers sufficiently short-ranged pair interactions, one obtains a partition of the interaction energy for a given site $i$ into contributions from confocal coordination spheres:

$$
U(i)=U_{I}(i)+U_{I I}(i)+\cdots+U_{K}(i)+\cdots .
$$

Usually, only the first neighboring shell of radius $R_{I}$ is considered. However, there are good reasons to keep the NNN shell of radius $R_{I I}$, too. In bcc crystals $R_{I I}: R_{I}=1: \sqrt{3} / 2$, i.e., there is only a slight increase of about $15 \%$ as one goes over from the first to the second coordination sphere. Additionally, in Sec. II we find that there is no symmetry breaking at bcc(100) and bcc(111) surfaces in the sense that the first nonvanishing contributions are of order $l=4$, as in the cubic bulk, and are not uniaxial-like of order $l=2$ as it turns out to be the case with the other surfaces we consider. In Sec. III, we extend the calculation for these two peculiar cases to account for NNN pair interactions as well. Accordingly, keeping the first two shells of a given site $i$,

$$
\begin{aligned}
U(i)= & U_{I}(i)+U_{I I}(i) \\
= & \frac{1}{2} \sum_{l} a_{l}\left(R_{I}\right)\left\{\sum_{j}^{I \text { shell }} P_{l}\left(\cos \alpha_{i j}\right)\right. \\
& \left.+\frac{a_{l}\left(R_{I I}\right)}{a_{l}\left(R_{I}\right)} \sum_{j}^{I I} \text { shell } P_{l}\left(\cos \alpha_{i j}\right)\right\} .
\end{aligned}
$$

Obviously, the price to be paid for getting more detailed information from Néel's pair model is the necessity to consider more interaction parameters $\left\{a_{l}\right\}$ that have to be independently determined by an atomistic theory and/or experiments. A useful estimate is that, for a given coordination sphere, the number of phenomenological parameters $\left\{a_{l}\right\}$ is less than, or equal to, the product of the number of considered orders in $l$ times the number of types of inequivalent site, and it should be kept in mind that the latter number itself depends on the coordination sphere considered. ${ }^{11}$ For example, for both bcc(100) and bcc(111) surfaces there is only one type of site with respect to the first (NN) shell and two types of site with respect to the second (NNN) shell. The contributions of these two types turn out to be identical (see
Sec. III), but this is certainly not a general property to be expected to hold in other geometries or to further coordination spheres. With fcc(110) surfaces, there are two inequivalent sites already with respect to the nearest shell.

In Eq. (6), in each order $l$ and in each coordination sphere the microscopic interaction represented by the $a_{l}$ 's has been separated from the purely structural part specified by the sum over the coordination angles $\alpha_{i j}$. Here we are not interested in the atomic origin of the $a_{l}$ 's but treat them as phenomenological parameters. There are, however, models such as the point-charge model ${ }^{13}$ and the screened-charge model ${ }^{14}$ that may be used to estimate Néel's parameters for a limited variety of magnetic materials (see Sec. III C 2). As a rule, for $R_{I}=2.5$ A the parameter $a_{2}\left(R_{I}\right)$ is of the order of $10^{-23} \mathrm{~J}$, whereas higher-order NN parameters are smaller by at least an order of magnitude. Note that Eq. (6) is not restricted to ideal crystals but applies also to edges, steps or local vacancies as well.

Another important problem of general character is the relation between Néel's model and phenomenological expansions such as the uniaxial expression

$$
\widetilde{U}=V\left(K_{1} \sin ^{2} \Theta+K_{2} \sin ^{4} \Theta+K_{3} \sin ^{6} \Theta\right),
$$

where $\Theta$ is the angle between the magnetization $\mathbf{M}$ and the axis of symmetry. Unfortunately, expansions of this type are neither orthogonal nor complete on the unit sphere of magnetization directions. ${ }^{15,16}$ In this work we shall restrict ourselves to orthonormal expansions ${ }^{10}$ into anisotropy coefficients. We shall use a number of low-indexed surfaces to derive explicit results and to demonstrate the predictive power of the method. The relation of the set of anisotropy coefficients to the set of anisotropy constants is discussed in Appendix C.

We wish to emphasize at this place that consideration of the symmetry breaking due to the often non-negligible strain and magnetoelastic effects lies beyond the scope of this paper. The inclusion of such effects into Néel's model should follow the lines suggested in Refs. 7 and 8.

\section{CALCULATION AND RESULTS}

In this section, we evaluate the contributions from the first (NN) coordination shell only [the first term in Eq. (6)]. The extension to the second (NNN) shell is straightforward and is presented in the next section for bcc(100) and bcc(111) surfaces.

\section{A. General aspects of the procedure}

To proceed with the expression from Eq. (6), one uses the addition theorem for Legendre functions: ${ }^{10}$

$$
\begin{aligned}
P_{l}\left(\cos \alpha_{i j}\right)= & P_{l}(\cos \Theta) P_{l}\left(\cos \theta_{i j}\right) \\
& +2 \sum_{m=1}^{l} \frac{(l-m) !}{(l+m) !} P_{l m}(\cos \Theta) P_{l m}\left(\cos \theta_{i j}\right) \\
& \times\left(\cos m \Phi \cos m \phi_{i j}+\sin m \Phi \sin m \phi_{i j}\right),
\end{aligned}
$$

where the angles [Fig. 1(b)] are specified by 


$$
\mathbf{M}=M\left(\sin \Theta \cos \Phi \mathbf{e}_{\mathbf{x}}+\sin \Theta \sin \Phi \mathbf{e}_{\mathbf{y}}+\cos \Theta \mathbf{e}_{\mathbf{z}}\right)
$$

and

$$
\mathbf{r}_{\mathbf{i j}}=r_{i j}\left(\sin \theta_{i j} \cos \phi_{i j} \mathbf{e}_{\mathbf{x}}+\sin \theta_{i j} \sin \phi_{i j} \mathbf{e}_{\mathbf{y}}+\cos \theta_{i j} \mathbf{e}_{\mathbf{z}}\right) .
$$

The $P_{l m}$ 's are the associated Legendre functions of the first kind, whereas $P_{l 0}(x) \equiv P_{l}(x)$ are the usual Legendre polynomials as introduced in Eq. (1) (cf. Appendix A). Inserting Eq. (8) into Eq. (6) yields, after a straightforward calculation,

$$
\begin{aligned}
U(i)= & \sum_{l} G_{l}(i) P_{l}(\cos \Theta) \\
& +\sum_{l} \sum_{m=1}^{l} G_{l m}^{(c)}(i) P_{l m}(\cos \Theta) \cos m \Phi \\
& +\sum_{l} \sum_{m=1}^{l} G_{l m}^{(s)}(i) P_{l m}(\cos \Theta) \sin m \Phi .
\end{aligned}
$$

The subscripts $(c)$ and $(s)$ stand for cosine and sine, respectively. All microscopic details about structure and interaction are contained in the quantities $G_{l m}(i)$ :

$$
\begin{aligned}
G_{l}(i) & =\frac{1}{2} a_{l}\left(R_{I}\right) S_{l}(i), \\
G_{l m}^{(c)}(i) & =a_{l}\left(R_{I}\right) \frac{(l-m) !}{(l+m) !} S_{l m}^{(c)}(i), \\
G_{l m}^{(s)}(i) & =a_{l}\left(R_{I}\right) \frac{(l-m) !}{(l+m) !} S_{l m}^{(s)}(i) .
\end{aligned}
$$

In the above, to facilitate computation and tabulation, we have introduced the proper structural sums $\left\{S_{l m}\right\}$ as

$$
\begin{aligned}
S_{l}(i) & =\sum_{j}^{I \text { shell }} P_{l}\left(\cos \theta_{i j}\right), \\
S_{l m}^{(c)}(i) & =\sum_{j}^{I \text { shell }} P_{l m}\left(\cos \theta_{i j}\right) \cos m \phi_{i j}, \\
S_{l m}^{(s)}(i) & =\sum_{j}^{I \text { shell }} P_{l m}\left(\cos \theta_{i j}\right) \sin m \phi_{i j} .
\end{aligned}
$$

Now, since the magnetization degrees of freedom $(\Theta, \Phi)$ have been factored out in Eq. (9), the energy per magnetic site from Eqs. (4) and (9) is

$$
\begin{aligned}
\frac{U}{N}= & \sum_{l} \kappa_{l} P_{l}(\cos \Theta)+\sum_{l} \sum_{m=1}^{l} \kappa_{l m}^{(c)} P_{l m}(\cos \Theta) \cos m \Phi \\
& +\sum_{l} \sum_{m=1}^{l} \kappa_{l m}^{(s)} P_{l m}(\cos \Theta) \sin m \Phi,
\end{aligned}
$$

where

$$
\kappa_{l} \equiv \sum_{\mu=1}^{p}\left(\frac{N_{\mu}}{N}\right) G_{l}(\mu)
$$

$$
\begin{gathered}
\kappa_{l m}^{(c)} \equiv \sum_{\mu=1}^{p}\left(\frac{N_{\mu}}{N}\right) G_{l m}^{(c)}(\mu), \\
\kappa_{l m}^{(s)} \equiv \sum_{\mu=1}^{p}\left(\frac{N_{\mu}}{N}\right) G_{l m}^{(s)}(\mu) .
\end{gathered}
$$

In other words, the Néel macroscopic anisotropy coefficients $\{\kappa\}$ are weighted averages of the site dependent, local anisotropy coefficients $\{G(\mu)\}(\mu=1,2, \ldots, p, p$ is the number of inequivalent types of site). ${ }^{11}$

\section{B. Results for bec and fec symmetries}

The pair-model anisotropies for body-centered-cubic (bcc) and face-centered-cubic (fcc) crystal symmetries follow from Eqs. (10)-(15) and (16)-(19). Here we consider ideal bulk crystals and their (100), (110), and (111) surfaces. More precisely, we derive the anisotropy energy $U(i)$ per magnetic site up to sixth order in $l$.

To this end, it only remains to evaluate for $l=2,4,6$ the structure factors $\{S\}$ and, hence, the local anisotropy coefficients $\{G\}$ in Eqs. (10)-(15) for $l=2,4,6$. The frame of reference is shown in Fig. 2; the polar $(z)$ axis is chosen outwards perpendicular to the surface. For comparison, the bulk structure factors have been computed in the same frames, i.e., the $z$ axis is perpendicular to the planes (100), (110), or (111), respectively. This means that for the bulk neighborhood one must additionally consider the sites that are mirror reflections of the hollow (below-the-surface) neighbors with respect to the surface in Fig. 2. The required structural information is presented in Appendix B; Tables I and II record the results for the surface and bulk cases, respectively.

At the surface (Table I), there are two distinct surface contributions in the fcc(110) case that are labeled $A$ and $B$ in the last two columns, i.e., a case per atom in the first and second layers, respectively. This can be recognized in Fig. 2 where each atom in the second layer misses a neighbor in the topmost layer. In the terminology of the introductory chapter, there are two inequivalent sites for fcc(110) surfaces already with respect to the $\mathrm{NN}$ shell.

In the bulk case (Table II), the prefactor of two in the first two columns, concerning bcc(100) and bcc(111), is used to stress the fact that there is a simple relation between the bulk and surface coefficients in the chosen frame of reference, namely, the anisotropy contribution of a bulk atom is twice that of a surface atom. This proportionality correlates with the absence of geometrical symmetry breaking in these cases, so that no second-order $(l=2)$ uniaxial anisotropies are generated at these surfaces. The quest for lowest-order symmetry-breaking contributions in the peculiar cases of bcc(100) and bcc(111) surfaces will be continued in the next section by considering the NNN pair interactions. In all other cases, considered in this section, the symmetry-breaking results in the emergence of uniaxial contributions ${ }^{17}$ and in the impossibility to reduce the difference between surface and bulk to a simple proportionality.

\section{Analysis of the results}

In Tables I and II, a nonzero azimuthal anisotropy contribution $S_{l m}$ whose index $m$ is not zero indicates that the re- 



$(\operatorname{bcc}(001)$

fcc $(001)$

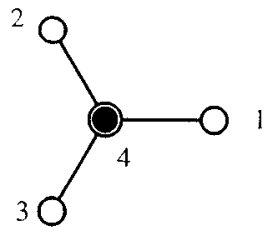

bec $(111)$

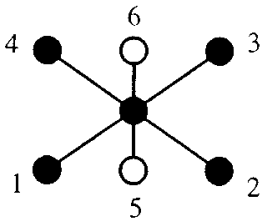

bec $(110)$
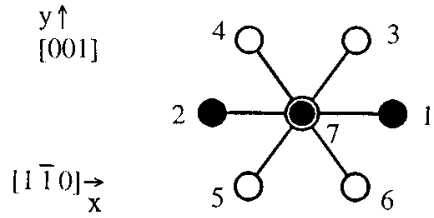

$\operatorname{lec}(110)$

FIG. 2. Atomic environment of surface atoms in cubic geometry. The $x$ and $y$ axes lie in the plane of the figure and are indicated by arrows. The $z$ axis is outwards perpendicular to the plane of the figure. Black circles denote sites at the surface, hollow circles stand for nearest neighbors below the surface. For the bulk calculation, in each case one has to consider also the mirror reflections of the hollow circles with respect to the surface.

spective $z$ axis is an $m$-fold symmetry axis for the overall anisotropy energy $U(\Theta, \Phi)$ of Eq. (16). Going down a given column, in any particular case one can easily recognize whether the azimuthal contribution is of the same order $m$ for all $l$. If this is the case, the eventual determination of the resultant easy axis of magnetization $\left(\Theta_{0}, \Phi_{0}\right)$ by minimization of $U$ [Eq. (16)] or $F_{A}$ [Eq. (22)] is trivial, since the resulting trigonometric equations for the two magnetization degrees of freedom $(\Theta, \Phi)$ are decoupled. By inspection, this is the case only for bcc(100) and fcc(100) where the $z$ axis is an axis of fourfold azimuthal symmetry. ${ }^{18,19}$ In all the other cases, there are different $m$ 's for different $l$ 's $(m \leqslant l)$, so that the minimization problem leads to untrivially coupled equations for the determination of the equilibrium values $\left(\Theta_{0}, \Phi_{0}\right)$. In the bulk, this situation is not entirely new and

related problems have been discussed. ${ }^{20}$ At the surface, however, with the exception of the no-symmetry-breaking cases bcc(100) and bcc(111) discussed above, one has to tackle minimization problems that are considerably more complicated than the corresponding bulk ones. For fcc(100) and fcc(111), the difficulty enters with the uniaxial $(l=2$, $m=0)$ term only, whereas the bcc(110) and fcc(110) cases involve a twofold azimuthal anisotropy contribution $(l=2$, $m=2)$ coupled to the uniaxial one $(l=2, m=0)$. Thus, the effective symmetry of the magnetic anisotropy energy is very low. This signals the existence of cases where the equilibrium magnetization is neither perpendicular nor parallel to the surface. Moreover, for such low-symmetry cases there would arise nontrivial thickness- or temperature-driven spin reorientation transitions in very thin ferromagnetic films where the surface contributions dominate. ${ }^{3,4,21}$ In these transitions, the magnetization would proceed from its initial easy axis to its final orientation without remaining in a fixed plane all along.

The restriction to nearest neighbors brings about the spectacular consequence that all possible ratios between the anisotropy coeficients of given order $l$ are independent of the microscopic energy parameters $\left\{a_{l}\right\}$. Note that this is true for both sets of local $(G)$ and total $(\kappa)$ anisotropy coefficients. For instance,

$$
\frac{G_{l m}^{(c)}(i)}{G_{l}(i)}=2 \frac{(l-m) !}{(l+m) !}\left[\frac{S_{l m}^{(c)}(i)}{S_{l}(i)}\right] .
$$

with the sums in the brackets given in Tables I and II. Such ratios have been used in experimental bulk magnetism to analyze anisotropy contributions ${ }^{22,23}$ and can be of interest in the context of thin-film and surface magnetism as well.

Since there are only even-order Néel contributions ( $l$ $=2 h$ ), the maximal number $n_{\text {max }}$ of total anisotropy coefficients $\left\{\kappa_{l m}\right\}$ is given by

$$
n_{\max }=h+2 \sum_{s=1}^{h}(2 s)=h(2 h+3) .
$$

Consequently, there are at most 5, 14, or 27 nonzero coefficients for $l=2,4$, or 6 , respectively. The same estimate holds for each species of local coefficient $\{G(\mu)\}$. In fact, there is some redundance in Eq. (21) and there exists a minimal set of coefficients $n_{\min }$ that is determined by the symmetry of the surroundings. Hence, what one finds in Table II are just two groups of anisotropy coefficients (and not six as the number of columns might suggest), one per bulk bcc and bulk fcc, respectively. Within each group, along each row in the table one finds the same bulk anisotropy coefficient, computed in three different frames of reference. ${ }^{24}$ The minimal number of coefficients $n_{\text {min }}$ to order $l=6$ equals four for both bec and fcc bulk magnets.

\section{DISCUSSION AND CONCLUSIONS}

\section{A. Atomistic origin of Néel's parameters}

A question of particular interest is the atomic origin of the pair-potential interaction parameters $\left\{a_{l}\right\}$. Two examples are the results for $a_{l}$ within the point-charge model ${ }^{13}$ and the screened-charge model. ${ }^{14}$ Both are based on the 
TABLE I. Structure factors $\{S\}$ for bcc and fcc surfaces in the frame of reference defined in Fig. 2. Cf. Eqs. (13)-(15). Only nearest-neighbor contributions are listed here.

\begin{tabular}{lccccccc}
\hline \hline$S_{l m}$ & $\mathrm{bcc}(100)$ & $\mathrm{bcc}(111)$ & $\mathrm{bcc}(110)$ & $\mathrm{fcc}(100)$ & $\mathrm{fcc}(111)$ & $\mathrm{fcc}(110)-A$ & $\mathrm{fcc}(110)-B$ \\
\hline$S_{2}$ & 0 & 0 & -1 & -1 & $-3 / 2$ & $-1 / 2$ & -1 \\
$S_{22}$ & 0 & 0 & 2 & 0 & 0 & 3 & 0 \\
\hline$S_{4}$ & $-14 / 9$ & $28 / 27$ & $41 / 36$ & $-1 / 8$ & $41 / 24$ & $19 / 32$ & $-9 / 16$ \\
$S_{42}^{(c)}$ & 0 & 0 & $-85 / 3$ & 0 & 0 & $-165 / 8$ & $-105 / 4$ \\
$S_{43}^{(c)}$ & 0 & $-560 \sqrt{2} / 9$ & 0 & 0 & 0 & 0 & 0 \\
$S_{43}^{(s)}$ & 0 & 0 & 0 & 0 & $-35 \sqrt{2} / 2$ & 0 & 0 \\
$S_{44}^{(s)}$ & $-560 / 3$ & 0 & $-910 / 3$ & -315 & 0 & $105 / 4$ & $-315 / 2$ \\
\hline$S_{6}$ & $8 / 9$ & $128 / 81$ & $-149 / 72$ & $-59 / 32$ & $-149 / 48 \sqrt{2} / 2$ & $427 / 256$ & $379 / 128$ \\
$S_{62}^{(c)}$ & 0 & 0 & $-525 / 36$ & 0 & 0 & $5775 / 128$ & $4095 / 64$ \\
$S_{63}^{(c)}$ & 0 & $4480 \sqrt{2} / 27$ & 0 & 0 & 0 & 0 & 0 \\
$S_{63}^{(s)}$ & 0 & 0 & 0 & 0 & $-455 \sqrt{2} / 2$ & 0 & 0 \\
$S_{64}^{(c)}$ & 2240 & 0 & 2135 & $16065 / 4$ & 0 & $-76545 / 32$ & $-61425 / 16$ \\
$S_{66}^{(c)}$ & 0 & $197120 / 9$ & -36190 & 0 & 61215 & $571725 / 16$ & $405405 / 8$ \\
\hline \hline
\end{tabular}

assumptions of rigid spin-orbit coupling in rare-earth ions and electrostatic crystal-field interactions. By comparing the point-charge and screened-charge anisotropy predictions with those of Néel's approach, one obtains the parameters $a_{2}(R)=-Q_{2}\left(e Q / 4 \pi \epsilon_{0}\right)\left(1 / R^{3}\right)$ or $a_{2}(R)$ $=-Q_{2}\left(e Q / 4 \pi \epsilon_{0}\right)\left(e^{-q R} / R^{3}\right)\left(1+q R+\frac{1}{3} q^{2} R^{2}\right)$, respectively. Here $R$ is the distance between the nearest neighbors, $Q$ is the crystal-field charge of the neighboring atom, $Q_{2}$ is the electrostatic quadrupole moment of the rare-earth atom, while $q \approx 2,3 \quad \mathrm{~A}^{-1}$ is the inverse Thomas-Fermi screening length. ${ }^{25}$ An extension of this approach to $3 d$ metals is given in Ref. 26.

\section{B. Relation to general anisotropy-energy expansions}

It is useful to compare Eq. (16) with the complete and orthonormal expansion of the magnetic anisotropy energy $F_{A}(\Theta, \Phi)$ into spherical harmonics that can be written as ${ }^{27}$

TABLE II. Bulk structure factors $\{S\}$ for bcc and fcc magnets computed in the same frame of reference (Fig. 2). To emphasize the choice of the $z$ axis, we use the Miller indexation. As in Table I, the coefficients that are identically zero for all cases have been omitted. Compare Eqs. (13)-(15). Only NN contributions are listed here.

\begin{tabular}{|c|c|c|c|c|c|c|}
\hline$S_{l m}$ & bcc (100) & $\operatorname{bcc}(111)$ & bcc(110) & fcc $(100)$ & fcc(111) & fcc(110) \\
\hline$S_{4}$ & $-2 \times(14 / 9)$ & $2 \times(28 / 27)$ & $7 / 9$ & $-7 / 4$ & $7 / 6$ & $7 / 16$ \\
\hline$S_{42}^{(c)}$ & 0 & 0 & $-140 / 3$ & 0 & 0 & $-105 / 4$ \\
\hline$S_{43}^{(c)}$ & 0 & $-2 \times(560 \sqrt{2} / 9)$ & 0 & 0 & 0 & 0 \\
\hline$S_{43}^{(s)}$ & 0 & 0 & 0 & 0 & $-70 \sqrt{2} / 2$ & 0 \\
\hline$S_{44}^{(c)}$ & $-2 \times(560 / 3)$ & 0 & -280 & -210 & 0 & $-315 / 2$ \\
\hline$S_{6}$ & $2 \times(8 / 9)$ & $2 \times(128 / 81)$ & $-26 / 9$ & $-39 / 16$ & $-13 / 3$ & $507 / 128$ \\
\hline$S_{62}^{(c)}$ & 0 & 0 & $-140 / 3$ & 0 & 0 & $4095 / 64$ \\
\hline$S_{63}^{(c)}$ & 0 & $2 \times(4480 \sqrt{2} / 27)$ & 0 & 0 & 0 & 0 \\
\hline$S_{63}^{(s)}$ & 0 & 0 & 0 & 0 & $-455 \sqrt{2}$ & 0 \\
\hline$S_{64}^{(c)}$ & $-2 \times 2240$ & 0 & 2800 & $12285 / 2$ & 0 & $-61425 / 16$ \\
\hline$S_{66}^{(c)}$ & 0 & $2 \times(197120 / 9)$ & -36190 & 0 & 60060 & $405405 / 8$ \\
\hline
\end{tabular}


TABLE III. Next-nearest-neighbor structure factors $\{\hat{\mathcal{S}}\}$ for bcc(100) and bcc(111) surfaces in the same frame of reference as for the NN calculations (Fig. 2). Compare Eq. (23). Structure factors that are zero in both cases have not been listed. Compare with the NN contributions of Table I.

\begin{tabular}{lcc}
\hline \hline$\hat{\mathcal{S}}_{l m}$ & $\mathrm{bcc}(100)$ & $\mathrm{bcc}(111)$ \\
\hline$\hat{\mathcal{S}}_{2}$ & -1 & 0 \\
\hline$\hat{\mathcal{S}}_{4}$ & $5 / 2$ & $-7 / 6$ \\
$\hat{\mathcal{S}}_{43}^{(c)}$ & 0 & $70 \sqrt{2}$ \\
$\hat{\mathcal{S}}_{44}^{(c)}$ & 420 & 0 \\
\hline$\hat{\mathcal{S}}_{6}$ & $-1 / 4$ & $2 / 3$ \\
$\hat{\mathcal{S}}_{63}^{(c)}$ & 0 & $70 \sqrt{2}$ \\
$\hat{\mathcal{S}}_{64}^{(c)}$ & -1890 & 0 \\
$\hat{\mathcal{S}}_{66}^{(c)}$ & 0 & 9240 \\
\hline \hline
\end{tabular}

$$
\begin{aligned}
\frac{F_{A}(\Theta, \Phi)}{N}= & \sum_{l} A_{l} P_{l}(\cos \Theta) \\
& +\sum_{l} \sum_{m=1}^{l} A_{l m} P_{l m}(\cos \Theta) \cos m \Phi \\
& +\sum_{l} \sum_{m=1}^{l} B_{l m} P_{l m}(\cos \Theta) \sin m \Phi .
\end{aligned}
$$

Here the quantities $A_{l}, A_{l m}, B_{l m}$ are the usual phenomenological anisotropy coefficients. Equation (22) is more general than Eq. (16), because it is not based on model assumptions. In other words, the $A_{l m}$ 's and $B_{l m}$ 's may be assumed to encompass both Néel and non-Néel contributions. This recognition is of a general character and provides a possibility for bridging the gap or interpolating between different theories of magnetic anisotropy.

Regardless of whether the anisotropy coefficients are introduced purely phenomenologically as in Eq. (22) or are derived starting from a microscopic model as in Eq. (16), their connection with the traditionally more standard anisotropy constants deserves to be clarified. Indeed, the point has already been discussed in different contexts, ${ }^{15,16}$ but specific considerations of implemented theoretical approach, normalizing conventions, choice of notation, or the fact that the usually assumed anisotropy-constant expansions are neither orthogonal nor complete, almost invariably generate some confusion. This is why we discuss the issue on an example in Appendix C.

\section{Next-nearest-neighbor contributions at bcc(100) and bec(111) surfaces}

The consideration in the previous section was restricted to NN Néel interactions that is a fair approximation in most cases. As we demonstrated above, notable exceptions are bcc(001) and bcc(111) surfaces, where the lowest-order NN contribution is zero by symmetry. In this case, which is also the bulk cubic case, the surface anisotropy constants encompass contributions from higher-order anisotropy coefficients (see Appendix C). It becomes a question of particular importance whether and to what extent the next-nearest-neighbor pair interactions would produce symmetry-breaking lowestorder contributions to the overall anisotropy. This is a problem of immediate practical impact, since the bcc NNN distance which we defined as $R_{I I}$ is larger by only about $15 \%$ than the NN distance $R_{I}$, so that despite the exponential decay of the Slater-Koster hopping integrals ${ }^{28}$ the atomistic NNN interactions are about half as strong as the NN interactions. On the other hand, in both cases of interest there are twice as many sites from among the NNN interacting surface sites than there are from among the NN interacting sites. In both cases, these are a topmost site plus a site from the first underlayer (cf. Fig. 2).

The required geometrical information is given in Appendix B. Note that in bcc geometry the next-nearest neighborhood has the simple-cubic $(s c)$ configuration. In the case bcc(100), there are five NNN's; in the case bcc(111), there are three NNN's. In both cases, geometrical inspection indicates that the additional NNN contribution from a firstunderlayer atom is identical with the contribution of a topmost atom (a black atom in Fig. 2). Thus, not only are there more contributions per unit surface, but they are of the same sign and magnitude; consequently, there is no way that they would compensate each other, if different from zero; thus the exponential decay of the interaction parameters mentioned above could be counterbalanced [see also Eq. (24) below].

Now, in analogy to Eqs. (13)-(15), the proper NNN structural sums $\{\hat{\mathcal{S}}\}$ are given by

$$
\hat{\mathcal{S}}_{l}(i)=\sum_{j}^{I I \text { shell }} P_{l}\left(\cos \theta_{i j}\right)
$$

and similarly for the $m$-dependent NNN sums.

In both cases, we work in the same frames of reference as in Sec. II. The results of the calculation for the NNN surface structural sums $\{\hat{\mathcal{S}}\}$ are presented in Table III and have to be compared with those for the NN surface sums $\{S\}$ in Table II.

For the bcc(100) surface, the symmetry breaking is purely uniaxial with the normal to the surface as the axis of symmetry. The fourth-order, fourfold contribution $(l=4, m=4)$ is of the cosine type, whereas with $\mathrm{NN}$ interactions, it is of the sine type $\left(\hat{\mathcal{S}}_{44}^{(c)}=420\right.$ vs $\left.S_{44}^{(s)}=-560 / 3\right)$. This is due to the coherent rotation of the second neighborhood by $\pi / 4$ around the $z$ axis.

For the bcc(111) surface, the most striking feature is the absence of lowest-order symmetry-breaking terms even when account is taken of the interactions with the second neighborhood. On top of that, there comes the observation that the NNN contributions to the leading fourth order ( $l$ $=4$ ) have the opposite sign to those of the NN contributions (compare the second columns of Tables VII and XI), 
whereby their magnitudes are comparable and there are twice as many $\mathrm{NNN}$ contributions per unit surface as there are $\mathrm{NN}$ ones. Thus, for the bcc(111) surface, the contributions to the overall anisotropy coefficients $\{\kappa\}$ of Eq. (17) with account for both NN and NNN coordination shells will be proportional to the expression

$$
a_{4}\left(R_{I}\right) S_{4 m}^{(c)}\left[1-2 \frac{a_{4}\left(1,15 R_{I}\right)}{a_{4}\left(R_{I}\right)} \frac{\left|\hat{\mathcal{S}}_{4 m}^{(c)}\right|}{\left|S_{4 m}^{(c)}\right|}\right](m=0,3)
$$

[For $m=0$, the superscript (c) is to be skipped.] The explicit calculations combined with the argumentation about the exponential decay of Néel's parameters $\left\{a_{l}\right\}$ with $R_{I I}$ very close to $R_{I}$ favor a conclusion that the factor in the brackets of Eq. (24) would possibly be close to zero. This means that NNN Néel's contributions to bcc(111) surface anisotropies suppress the overall surface anisotropy in two spectacular ways. First, they do not break the symmetry and, consequently, the fourth-order terms $(l=4)$ remain dominant. Second, to this leading order they systematically oppose the NN contributions in sign; a more precise quantification of the implied reduction could come from a reliable atomistic estimate of $a_{4}(R)$.

\section{Summary}

We have extended Néel's model in two ways. First, we have used Néel's assumptions to calculate contributions to sixth order for low-index surfaces and for the bulk in cubic geometry. On the basis of the results given in Tables I and II for the surface and bulk contributions, respectively, it is possible to identify cases of very low angular symmetry. This low symmetry gives rise to complicated minimization problems for the determination of the equilibrium orientation of magnetization with or without an applied magnetic field and can lead to spin-reorientation transitions during which the magnetization vector does not remain in a fixed plane. Applications to nonideal materials are straightforward, since the approach is not restricted to ideal bulk materials and surfaces, but applies to vacancies, steps, and edges as well. Second, the contributions to the surface anisotropy from the second neighborhood have been examined for those cases [bcc(100) and bcc(111)] where the NN interactions do not suffice to break the symmetry (Table III). We find that NNN interactions do induce lowest-order contributions to bcc(100) surfaces and that, in contrast, the bcc(111) surface does not generate anisotropy contributions of the lowest order even with the second neighborhood taken into account. In this latter case, we detect a characteristic alternation of signs between NN and NNN leading $(l=4)$ contributions to the same anisotropy coefficients. In view of the proximity of the first- and second-shell radii in bcc geometry, this suggests a well-pronounced compensation effect as a result of the competition between NN and NNN pair interactions.

An important advantage of the present approach is the systematic use of the anisotropy coefficients, arising naturally in a multipole expansion of the anisotropy energy that is both complete and orthonormal. The careful partition of contributions into nonequivalent species of site, interaction shell, and order in the multipole expansion reveals, among other things, the constitution of the macroscopic anisotropy coefficients $\{\kappa\}$ as weighted averages of the local coefficients $\{G\}$ [Eqs. (17)-(19)]. On the other hand, the connection between the coefficients $\{\kappa\}$ and the more usual constants $\{K\}$ has been elucidated with emphasis on the advantages offered by the complete orthonormal set of anisotropy coefficients $\{\kappa\}$.

It is certainly an important and challenging experimental problem to find a suitable setting for the acquisition of sufficient information that would allow one to determine the higher-order Néel anisotropies reported here. Such information would shed light onto the nature and magnitude of higher-order anisotropy contributions. These are of special interest in magnetic systems undergoing spin reorientation transitions under variation of temperature or, in ultrathin films, of thickness. They are precisely the higher-order anisotropies that control and stabilize the behavior of the respective systems, since the leading-order contributions cancel at such a transition point.

\section{ACKNOWLEDGMENTS}

The authors appreciate valuable discussions with $\mathrm{H}$. P. Oepen.

\section{APPENDIX A}

In order to calculate the local anisotropy coefficients $\left\{G_{l m}\right\}$ of Eqs. (10)-(12), one needs two types of information. The analytical information is given here, while Appendix B presents the required stereometrical information.

The usual Legendre polynomials $P_{l}(x) \equiv P_{l 0}(x)$ can be found in quite a number of handbooks (see, e.g., Ref. 10). We need only those with $l=2,4,6$. They read

$$
\begin{gathered}
P_{2}(x)=\frac{1}{2}\left(3 x^{2}-1\right), \\
P_{4}(x)=\frac{1}{8}\left(35 x^{4}-30 x^{2}+3\right), \\
P_{6}(x)=\frac{1}{16}\left(231 x^{6}-315 x^{4}+105 x^{2}-5\right) .
\end{gathered}
$$

The associated Legendre functions $P_{l m}(x)$ are defined as

$$
P_{l m}(x)=\left(1-x^{2}\right)^{m / 2} d^{m} P_{l}(x) / d x^{m} .
$$

For even values of $m$, the $P_{l m}$ 's themselves reduce to polynomials. For any given $l$, we need all functions with $m \leqslant l$ :

$$
\begin{gathered}
P_{21}(x)=3 x\left(1-x^{2}\right)^{1 / 2}, \\
P_{22}(x)=3\left(1-x^{2}\right) ; \\
P_{41}(x)=\frac{5}{2} x\left(7 x^{2}-3\right)\left(1-x^{2}\right)^{1 / 2},
\end{gathered}
$$




$$
\begin{gathered}
P_{42}(x)=\frac{15}{2}\left(1-x^{2}\right)\left(7 x^{2}-1\right), \\
P_{43}(x)=105 x\left(1-x^{2}\right)^{3 / 2}, \\
P_{44}(x)=105\left(1-x^{2}\right)^{2} ; \\
P_{61}(x)=\frac{3}{8} x\left(231 x^{4}-210 x^{2}+35\right)\left(1-x^{2}\right)^{1 / 2}, \\
P_{62}(x)=\frac{105}{8}\left(1-x^{2}\right)\left(33 x^{4}-18 x^{2}+1\right), \\
P_{63}(x)=\frac{315}{2} x\left(11 x^{2}-3\right)\left(1-x^{2}\right)^{3 / 2}, \\
P_{66}(x)=10395\left(1-x^{2}\right)^{3} . \\
P_{64}(x)=\frac{945}{2}\left(11 x^{2}-1\right)\left(1-x^{2}\right)^{2}, \\
P_{65}(x)=10395 x\left(1-x^{2}\right)^{5 / 2},
\end{gathered}
$$

\section{APPENDIX B}

Here we summarize the required information about the morphology of the surroundings of a given site at the surface in cubic geometry (cf. also Fig. 2). The information about the first (NN) shell is collected in Tables IV-IX. To avoid complicated pictures, the NNN surface atoms are not given in Fig. 2. Their locations are defined in Tables $X$ and XI together with the additional information that the NNN coordination in bcc geometry is of the simple-cubic ( $s c$ ) type. For an atom in the respective cubic bulk, one has to add the missing neighbors as explained in the main text. The azimuthal coordinates $\{\phi\}$ of the added atoms are the same as for the hollow atoms in Fig. 2, while the polar coordinates $\{\theta\}$ complement to $\pi$ the $\theta$ 's of the hollow atoms.

In the calculation of $\left\{S_{l m}\right\}$ [Eqs. (13)-(15)], it is understood that the origin is at the $i$ th atom. Hence, we drop the index $i$ and let the index $j$ run over the nearest neigbors. Their number is denoted by $z_{N N}$. The same holds for the calculation of $\left\{\hat{\mathcal{S}}_{l m}\right\}$ with the number of NNN denoted by $z_{N N N}$.

The angles $\phi_{4}$ in Table $\mathrm{V}$ and $\phi_{7}$ in Table IX are labeled as indeterminate. This is because the corresponding neighbor lies on the $z$ axis below the central atom (Fig. 2). This indeterminacy is, however, irrelevant, since the respective $\theta$-dependent factors in the analytical expression vanish identically. The factors $\cos m \phi_{j}$ and $\sin m \phi_{j}$ encountered in the

TABLE IV. The surface $\operatorname{bcc}(001)\left(z_{\mathrm{NN}}=4\right)$.

\begin{tabular}{lccccc}
\hline \hline$j=1, \ldots, z_{\mathrm{NN}}$ & $\theta_{j} \in[0, \pi]$ & $\cos \theta_{j}$ & $\phi_{j} \in[0,2 \pi]$ & $\cos \phi_{j}$ & $\sin \phi_{j}$ \\
\hline 1 & $\arccos (-1 / \sqrt{3})$ & $-1 / \sqrt{3}$ & $\pi / 4$ & $1 / \sqrt{2}$ & $1 / \sqrt{2}$ \\
2 & $\arccos (-1 / \sqrt{3})$ & $-1 / \sqrt{3}$ & $3 \pi / 4$ & $-1 / \sqrt{2}$ & $1 / \sqrt{2}$ \\
3 & $\arccos (-1 / \sqrt{3})$ & $-1 / \sqrt{3}$ & $5 \pi / 4$ & $-1 / \sqrt{2}$ & $-1 / \sqrt{2}$ \\
4 & $\arccos (-1 / \sqrt{3})$ & $-1 / \sqrt{3}$ & $7 \pi / 4$ & $1 / \sqrt{2}$ & $-1 / \sqrt{2}$ \\
\hline \hline
\end{tabular}

TABLE V. The surface bcc(111) $\left(z_{\mathrm{NN}}=4\right)$.

\begin{tabular}{cccccc}
\hline \hline$j=1, \ldots, z_{\mathrm{NN}}$ & $\theta_{j} \in[0, \pi]$ & $\cos \theta_{j}$ & $\phi_{j} \in[0,2 \pi]$ & $\cos \phi_{j}$ & $\sin \phi_{j}$ \\
\hline 1 & $\arccos (-1 / 3)$ & $-1 / 3$ & 0 & 1 & 0 \\
2 & $\arccos (-1 / 3)$ & $-1 / 3$ & $2 \pi / 3$ & $-1 / 2$ & $\sqrt{3} / 2$ \\
3 & $\arccos (-1 / 3)$ & $-1 / 3$ & $4 \pi / 3$ & $-1 / 2$ & $-\sqrt{3} / 2$ \\
\hline 4 & $\pi$ & -1 & Indeterminate & & \\
\hline \hline
\end{tabular}

TABLE VI. The surface bcc(110) $\left(z_{\mathrm{NN}}=6\right)$.

\begin{tabular}{cccccc}
\hline \hline$j=1, \ldots, z_{\mathrm{NN}}$ & $\theta_{j} \in[0, \pi]$ & $\cos \theta_{j}$ & $\phi_{j} \in[0,2 \pi]$ & $\cos \phi_{j}$ & $\sin \phi_{j}$ \\
\hline 1 & $\pi / 2$ & 0 & $\phi_{1}=\pi+\phi_{3}$ & $-\sqrt{2 / 3}$ & $-1 / \sqrt{3}$ \\
2 & $\pi / 2$ & 0 & $\phi_{2}=2 \pi-\phi_{3}$ & $\sqrt{2 / 3}$ & $-1 / \sqrt{3}$ \\
3 & $\pi / 2$ & 0 & $\phi_{3}=0.18591 \pi$ & $\sqrt{2 / 3}$ & $1 / \sqrt{3}$ \\
4 & $\pi / 2$ & 0 & $\phi_{4}=\pi-\phi_{3}$ & $-\sqrt{2 / 3}$ & $1 / \sqrt{3}$ \\
\hline & & & & \\
5 & $\arccos (-\sqrt{2 / 3})$ & $-\sqrt{2 / 3}$ & $3 \pi / 2$ & 0 & -1 \\
6 & $\arccos (-\sqrt{2 / 3})$ & $-\sqrt{2 / 3}$ & $\pi / 2$ & 0 & 1 \\
\hline \hline
\end{tabular}


TABLE VII. The surface fcc $(001)\left(z_{\mathrm{NN}}=8\right)$.

\begin{tabular}{lccccc}
\hline \hline$j=1, \ldots, z_{\mathrm{NN}}$ & $\theta_{j} \in[0, \pi]$ & $\cos \theta_{j}$ & $\phi_{j} \in[0,2 \pi]$ & $\cos \phi_{j}$ & $\sin \phi_{j}$ \\
\hline 1 & $\pi / 2$ & 0 & $\pi / 4$ & $1 / \sqrt{2}$ & $1 / \sqrt{2}$ \\
2 & $\pi / 2$ & 0 & $3 \pi / 4$ & $-1 / \sqrt{2}$ & $1 / \sqrt{2}$ \\
3 & $\pi / 2$ & 0 & $5 \pi / 4$ & $-1 / \sqrt{2}$ & $-1 / \sqrt{2}$ \\
4 & $\pi / 2$ & 0 & $7 \pi / 4$ & $1 / \sqrt{2}$ & $-1 / \sqrt{2}$ \\
\hline 5 & $3 \pi / 4$ & $-1 / \sqrt{2}$ & 0 & 1 & 0 \\
6 & $3 \pi / 4$ & $-1 / \sqrt{2}$ & $\pi / 2$ & 0 & 1 \\
7 & $3 \pi / 4$ & $-1 / \sqrt{2}$ & $\pi$ & -1 & 0 \\
8 & $3 \pi / 4$ & $-1 / \sqrt{2}$ & $3 \pi / 2$ & 0 & -1 \\
\hline \hline
\end{tabular}

TABLE VIII. The surface fcc(111) $\left(z_{\mathrm{NN}}=9\right)$.

\begin{tabular}{cccccc}
\hline \hline$j=1, \ldots, z_{\mathrm{NN}}$ & $\theta_{j} \in[0, \pi]$ & $\cos \theta_{j}$ & $\phi_{j} \in[0,2 \pi]$ & $\cos \phi_{j}$ & $\sin \phi_{j}$ \\
\hline 1 & $\pi / 2$ & 0 & 0 & 1 & 0 \\
2 & $\pi / 2$ & 0 & $\pi / 3$ & $1 / 2$ & $\sqrt{3} / 2$ \\
3 & $\pi / 2$ & 0 & $2 \pi / 3$ & $-1 / 2$ & $\sqrt{3} / 2$ \\
4 & $\pi / 2$ & 0 & $3 \pi / 3$ & -1 & 0 \\
5 & $\pi / 2$ & 0 & $4 \pi / 3$ & $-1 / 2$ & $-\sqrt{3} / 2$ \\
6 & $\pi / 2$ & 0 & $5 \pi / 3$ & $1 / 2$ & $-\sqrt{3} / 2$ \\
\hline 7 & $\arccos (-\sqrt{2 / 3})$ & $-\sqrt{2 / 3}$ & $\pi / 6$ & $\sqrt{3} / 2$ & $1 / 2$ \\
8 & $\arccos (-\sqrt{2 / 3})$ & $-\sqrt{2 / 3}$ & $5 \pi / 6$ & $-\sqrt{3} / 2$ & $1 / 2$ \\
9 & $\arccos (-\sqrt{2 / 3})$ & $-\sqrt{2 / 3}$ & $9 \pi / 6$ & 0 & -1 \\
\hline \hline
\end{tabular}

TABLE IX. The surface fcc(110) $\left(z_{\mathrm{NN}}=7\right)$.

\begin{tabular}{cccccc}
\hline \hline$j=1, \ldots, z_{\mathrm{NN}}$ & $\theta_{j} \in[0, \pi]$ & $\cos \theta_{j}$ & $\phi_{j} \in[0,2 \pi]$ & $\cos \phi_{j}$ & $\sin \phi_{j}$ \\
\hline 1 & $\pi / 2$ & 0 & 0 & 1 & 0 \\
2 & $\pi / 2$ & 0 & $\pi$ & -1 & 0 \\
\hline 3 & $2 \pi / 3$ & $-1 / 2$ & $\pi / 3$ & $1 / 2$ & $\sqrt{3} / 2$ \\
4 & $2 \pi / 3$ & $-1 / 2$ & $2 \pi / 3$ & $-1 / 2$ & $\sqrt{3} / 2$ \\
5 & $2 \pi / 3$ & $-1 / 2$ & $4 \pi / 3$ & $1 / 2$ & $-\sqrt{3} / 2$ \\
6 & $2 \pi / 3$ & $-1 / 2$ & $5 \pi / 3$ & & $-\sqrt{3} / 2$ \\
\hline 7 & $\pi$ & -1 & Indeterminate & & \\
\hline
\end{tabular}

TABLE X. Next-nearest neighbors for the surface bcc $(100)\left(z_{\mathrm{NNN}}=5\right)$. The topmost (black) atom and the underlayer (hollow) atoms in Fig. 2 have an identical second coordination and, hence, acquire identical NNN Néel contributions. The NNN atoms are not given in Fig. 2; their spherical-coordinate angles are labeled with a " $I I$ " superscript.

\begin{tabular}{lccccc}
\hline \hline$j=1, \ldots, z_{N N N}$ & $\theta_{j}^{(I I)} \in[0, \pi]$ & $\cos \theta_{j}^{(I I)}$ & $\phi_{j}^{(I I)} \in[0,2 \pi]$ & $\cos \phi_{j}^{(I I)}$ & $\sin \phi_{j}^{(I I)}$ \\
\hline 1 & $\pi / 2$ & 0 & 0 & 1 & 0 \\
2 & $\pi / 2$ & 0 & $\pi / 2$ & 0 & 1 \\
3 & $\pi / 2$ & 0 & $\pi$ & -1 & 0 \\
4 & $\pi / 2$ & 0 & $3 \pi / 2$ & 0 & -1 \\
\hline 5 & $\pi$ & -1 & Indeterminate & & \\
\hline \hline
\end{tabular}


TABLE XI. Next-nearest neighbors for the surface bcc(111) $\left(z_{\mathrm{NNN}}=3\right)$. The topmost (black) atom and the underlayer (hollow) atoms in Fig. 2 have an identical second coordination and, hence, acquire identical NNN Néel contributions. The NNN atoms are not given in Fig. 2; their spherical-coordinate angles are labeled with a " $I I$ '" superscript.

\begin{tabular}{lccccc}
\hline \hline$j=1, \ldots, z_{\mathrm{NNN}}$ & $\theta_{j}^{(I I)} \in[0, \pi]$ & $\cos \theta_{j}^{(I I)}$ & $\phi_{j}^{(I I)} \in[0,2 \pi]$ & $\cos \phi_{j}^{(I I)}$ & $\sin \phi_{j}^{(I I)}$ \\
\hline 1 & $\arccos (-1 / \sqrt{3})$ & $-1 / \sqrt{3}$ & $\pi / 3$ & $1 / 2$ & $\sqrt{3} / 2$ \\
2 & $\arccos (-1 / \sqrt{3})$ & $-1 / \sqrt{3}$ & $\pi$ & -1 & 0 \\
3 & $\arccos (-1 / \sqrt{3})$ & $-1 / \sqrt{3}$ & $5 \pi / 3$ & $1 / 2$ & $-\sqrt{3} / 2$ \\
\hline \hline
\end{tabular}

calculation may be found either by direct computation with the respective value of $\phi_{j}$ or by first reducing them to $\cos \phi_{j}$ and $\sin \phi_{j}$ and using the last two columns in Tables IV-IX.

\section{APPENDIX C}

To stimulate the eventual application of our results to specific cases, we comment on the simple recipe to establish the quantitative connection between anisotropy coefficients and anisotropy constants.

One starts with the anisotropy-coefficient expansion [Eq. (16)] and terminates it to such a value of $l\left(l_{\max }=2 q_{\max }\right)$ that corresponds to the highest power of $\sin \Theta$ or $\cos \Theta$, appearing in the anisotropy-constant expansion with which we would like to establish correspondence. This done and with the explicit expressions for $P_{l}(\cos \Theta)$ and $P_{l m}(\cos \Theta)$ given in Appendix A, one has to just collect all terms in Eq. (16) which have the appearance of a given term in the anisotropy-constant expansion. As an example, if the anisotropy-constant energy density for uniaxial (cylindrical) symmetry is employed, one has

$$
\tilde{U} / V=K_{1} \sin ^{2} \Theta+K_{2} \sin ^{4} \Theta+K_{3} \sin ^{6} \Theta,
$$

where $\Theta$ is the angle between $\mathbf{M}$ and the axis of symmetry (this implies that the $z$ axis coincides with the $c$-axis). Since the structure of the crystal is assumed to be known, it should present no difficulty to convert the anisotropy energy density $\widetilde{U} / V$ to anisotropy energy per magnetic site by inserting the volume per magnetic site $v_{0} \equiv V / N$. In Eq. (C1), the azimuthal dependence is dropped. Physically, this is equivalent to neglecting anisotropies in the plane perpendicular to the $c$ axis. Accordingly, only the sum with $m=0$ has to be considered in the anisotropy-coefficent expansion of Eq. (16) and this is $U / N=\sum_{l=2,4,6} \kappa_{l} P_{l}(\cos \Theta)$. Collecting in turn terms in this sum which are proportional to $\sin ^{2} \Theta, \sin ^{4} \Theta$, and $\sin ^{6} \Theta$, one gets

$$
\begin{gathered}
v_{0} K_{1}=-\frac{3}{2} \kappa_{2}-5 \kappa_{4}-\frac{21}{2} \kappa_{6}, \\
v_{0} K_{2}=\frac{35}{8} \kappa_{4}+\frac{189}{8} \kappa_{6}, \\
v_{0} K_{3}=-\frac{231}{16} \kappa_{6} .
\end{gathered}
$$

The cases with nonzero in-plane contributions $(m \neq 0)$ are treated along the same lines.
*On leave from the CPCS Lab, Institute of Solid State Physics, Bulgarian Academy of Sciences, 1784 Sofia, Bulgaria.

${ }^{1}$ L. Néel, J. Phys. Radium 15, 225 (1954).

${ }^{2}$ P. Bruno, J. Phys. F 18, 1291 (1988); Phys. Rev. B 39, 865 (1989)

${ }^{3}$ B. Heinrich and J. F. Cochran, Adv. Phys. 42, 523 (1993).

${ }^{4}$ U. Gradmann, in Handbook of Magnetic Materials, edited by K. H. J. Buschow (Elsevier, Amsterdam, 1993), Vol. 7, pp. 1-95.

${ }^{5}$ P. Krams, B. Hillebrands, G. Güntherodt, and H. P. Oepen, Phys. Rev. B 49, 3633 (1994).

${ }^{6}$ D. S. Chuang, C. A. Ballentine, and R. C. O'Handley, Phys. Rev. B 49, 15084 (1994).

${ }^{7}$ O. Song, C. A. Ballentine, and R. C. O'Handley, Appl. Phys. Lett. 64, 2693 (1994).

${ }^{8}$ R. H. Victora and J. M. MacLaren, J. Appl. Phys. 73, 6415 (1993); Phys. Rev. B 47, 11583 (1993).

${ }^{9}$ G. H. O. Daalderop, P. J. Kelly, and M. F. H. Schuurmans, Phys. Rev. B 42, 7270 (1990).

${ }^{10}$ E. Jahnke, F. Emde, and F. Lösch, Tables of Special Functions (Teubner, Leipzig, 1960).

${ }^{11}$ Sites may be crystallographically or chemically inequivalent. To keep the analysis within tolerable limits, we do not examine the latter type of inequivalence.
${ }^{12}$ Even at this general level and before explicit calculations have been actually done, overall geometric considerations may reveal important qualitative features of the distinct contributions. If, e.g., $N_{1}$ equivalent sites are on a surface of a plate of dimensions $L \times L \times d$, then $N_{1} \sim S=L^{2}$, while $N \sim V=L^{2} d$ that brings about $N_{1} / N \sim 1 / d$. Hence, such a contribution can be neglected for a very thick plate $(d \rightarrow \infty)$, but will dominate in the ultrathin limit. This is then the usually assumed $1 / d$ contribution to the overall anisotropy. An example of similar counting can be found in Ref. 6 where nearest-neighbor interactions are considered and there are as many as four different species that are identified as clusters. For the validity of the above reasoning, it is sufficient that the pair interactions are short ranged.

${ }^{13}$ M. T. Hutchings, Solid State Phys. 16, 227 (1964).

${ }^{14}$ R. Skomski, Philos. Mag. B 70, 175 (1994).

${ }^{15}$ E. Callen and H. Callen, Phys. Rev. 139, A455 (1965); R. R. Birss, in Selected Topics in Solid State Physics, edited by E. P. Wohlfarth (North-Holland, Amsterdam, 1966), Vol. III; R. R. Birss and G. J. Keeler, Phys. Status Solidi B 64, 357 (1974); P.-A. Lindgard and O. Danielsen, Phys. Rev. B 11, 351 (1975).

${ }^{16}$ The complete and orthonormal expansion in anisotropy coefficients arises as the more fundamental one also in the analysis of 
the temperature dependence of anisotropy by statisticalmechanical means [E. R. Callen and H. B. Callen, J. Phys. Chem. Solids 27, 1271 (1966); Y. Millev and M. Fähnle, Phys. Rev. B 52, 4336 (1995)].

${ }^{17}$ There still might be some accidental degeneration at the microscopic level that could lead to $a_{2}=0$.

${ }^{18}$ Bulk uniaxial crystals of tetragonal or hexagonal symmetry belong mathematically to the same simple case with $m=4$ and 6 , respectively.

${ }^{19}$ M. Brockmann, S. Miethaner, R. Onderka, K. Köhler, F. H. Himmelhuber, H. Regensburger, F. Bensch, T. Schweinböck, and G. Bayreuther, J. Appl. Phys. 81, 5047 (1997).

${ }^{20}$ D. Krause, Phys. Status Solidi 6, 129 (1964); J. P. Rebouillat, J. Phys. (France) 32, C1-547 (1971); G. Asti and S. Rinaldi, J. Appl. Phys. 45, 3600 (1974); J. R. Cullen and E. Callen, Phys. Rev. B 30, 181 (1984).

${ }^{21}$ Y. Millev and J. Kirschner, Phys. Rev. B 54, 4137 (1996).

${ }^{22}$ D. J. Newman and B. Ng, Rep. Prog. Phys. 52, 699 (1989).

${ }^{23}$ J. M. D. Coey, in Science and Technology of Nanostructured Magnetic Materials, edited by G. C. Hadjipanayis and G. A. Prinz (Plenum, New York, 1991), p. 439.

${ }^{24}$ This is an explicit demonstration of the covariance of the anisotropy coefficients. The energy per site $U(i)$ is invariant as can be seen from Eq. (3) that depends only on the relative quantities involving $\mathbf{M}$ and $\mathbf{r}_{\mathbf{j}}-\mathbf{r}_{\mathbf{i}}$, but not on the frame of reference. Denoting with primes the quantities in a new, rotated frame, the above means that $U(i)=U^{\prime}(i)$ as it must be for the interaction energy. On the other hand, the multipole-expansion theorem guarantees that $U^{\prime}(i)$ has an expansion of the same form as $U(i)$ in Eq. (10) with $\Theta \rightarrow \Theta^{\prime}$ and $\Phi \rightarrow \Phi^{\prime}$. To ensure that $U(i)=U^{\prime}(i)$, one must reckon with $G^{\prime} \neq G$, in principle. The $\{G\}^{\prime}$ 's can be computed directly with the respective $\theta_{i j}^{\prime}$ and $\phi_{i j}^{\prime}$ instead of $\theta_{i j}$ and $\phi_{i j}$ or can be derived from the set $\{G\}$, if this has already been found in the initial frame of reference, by an appropriate rotation $\hat{R}(\alpha)$ that can be parametrized by the respective Euler angles.

${ }^{25}$ S. Wirth, M. Wolf, K.-H. Müller, R. Skomski, S. Brennan, and J. M. D. Coey, IEEE Trans. Magn. 32, 4746 (1996).

${ }^{26}$ R. Skomski, IEEE Trans. Magn. 32, 4794 (1996).

${ }^{27}$ The convergence of the multipole expansion is relevant to the problem of how many anisotropy coefficients and, hence, anisotropy constants would be needed for a proper description of $U(\Theta, \Phi)$. General spin-orbit-ineraction arguments [L. D. Landau and E. M. Lifshitz, Electrodynamics of Continuous Media (Pergamon, Oxford, 1960), Chap. 5] as well as estimates of the energetic parameters involved in particular systems (Refs. 1 and 6) speak out for a very rapid convergence. Furthermore, in systems where the magnetic anisotropy is due to rare-earth $(4 f)$ ions and the mixing of $J$ multiplets can be ignored, there are only the $l=2,4,6$ contributions [R. J. Elliott, in Magnetic Properties of Rare-Earth Metals, edited by R. J. Elliott (Plenum, New York, 1972), Chap. 1, pp. 1-16]. Still, peculiarities in the sense of poor convergence cannot be ruled out, for example, in itinerant ferromagnets.

${ }^{28}$ J. C. Slater and G. F. Koster, Phys. Rev. 94, 1498 (1954). 